\title{
Anesthetic Management of Aortopexy, Posterior Tracheopexy and Esophageal Atresia Repair in a Preterm Newborn
}

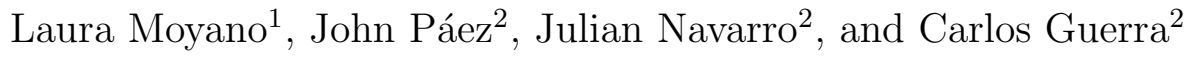 \\ ${ }^{1}$ Fundación Hospital Pediátrico La Misericordia \\ ${ }^{2}$ Fundación Hospital Pediátrico La Misericordia
}

February 15, 2022

\begin{abstract}
A 35-week neonate with esophageal atresia, tracheoesophageal fistula and tracheomalacia of was taken to a complex repair using bronchoscopic guidance. Invasive monitors were employed considering the length procedure and postoperative needs. Ventilation settings varied to maintain an adequate ventilation and surgical field. He was soon extubated in the ICU.
\end{abstract}

\section{Anesthetic Management of Aortopexy, Posterior Tracheopexy and Esophageal Atresia Repair in a Preterm Newborn.}

\begin{abstract}
Severe tracheobronchomalacia is usually associated with syndromes which make the management essentially surgical and challenging. A 35-week neonate with esophageal atresia, tracheoesophageal fistula and tracheomalacia of approximately $40 \%$ was taken to an aortopexy, tracheopexy and esophageal atresia repair using bronchoscopic guidance. Total intravenous anesthesia (TIVA) was used. A non-cuffed endotracheal tube that allowed the use of the fibrebronchoscope and the Arndt multiport airway adapter was selected. Standard and invasive monitors were employed taking into account the length procedure and postoperative needs. Ventilation settings varied to maintain an adequate ventilation and surgical field manipulation. He remained hemodynamically stable during the procedure and was soon extubated in the ICU.
\end{abstract}

Key words: Tracheobronchomalacia; Esophageal Atresia; Newborn.

Case Presentation: A 35-week neonate with esophageal atresia and tracheoesophageal fistula corrected by thoracoscopy presented persistent atelectasis and failed extubations, which led to a high suspicion of tracheomalacia and relapsing fistula. A bronchoscopy revealed tracheomalacia of approximately $40 \%$. A multidisciplinary group decided an aortopexy, tracheopexy and esophageal atresia repair. The preoperative echocardiogram showed a non-hemodynamically significant ductus arteriosus and adequate biventricular function. Preoperative endoscopic airway evaluation was performed under general anesthesia in a spontaneously breathing patient, it showed severe tracheobronchomalacia with a $70 \%$ dynamic collapse of the posterior aspect of the trachea as showed in Figure 1, based on this finding and the need to repair the esophageal atresia, a right posterior thoracotomy was decided. Pexy procedures were done using bronchoscopic guidance. Total intravenous anesthesia (TIVA) with Propofol-remifentanil infusions and hourly cisatracurium were used. A non-cuffed endotracheal tube \#4 was used, which allowed the use of the fiberoptic bronchoscope. Due to the patient's size lung isolation, one-lung ventilation and recurrent laryngeal nerve monitor were not used; still an Arndt multiport airway adapter was used to control the flexible fiberoptic bronchoscope, suction and ventilate at the same time. Standard monitors were used, also pre-ductal and post-ductal arterial lines (AL) to identify any changes secondary to obstruction or traction of the brachiocephalic trunk during the aortopexy. Bilateral internal jugular vein accesses were used considering the 
vasopressor and parenteral nutrition need. An epinephrine infusion was used throughout the procedure and titrated according to patient requirements. Endoluminal bronchoscopy was used to assist suturing the posterior membranous trachea to the anterior spinal ligament. Ventilation settings varied through the procedure to maintain an adequate ventilation, adequate surgical field view and manipulation; a pressure-controlled ventilation mode with maximal airway pressures of 20-25 $\mathrm{cmH} 2 \mathrm{O}$, respiratory rate 20-30 rpm and PEEP 5, FiO2 50-80\% was used. He remained hemodynamically stable during the procedure and was extubated in the ICU within 12 hours.

Shallow Breathing - 31.08.20

\begin{tabular}{lll}
\hline Anatomic Divisions & PreOP Collapse/Pressure & PostOP Collapse/Pressure \\
\hline L3 - left mainstem bronchus level 3 & $40 \%-50 \mathrm{mmHg}$ & $20 \%-50 \mathrm{mmHg}$ \\
L2 - left mainstem bronchus level 2 & $70 \%-30 \mathrm{mmHg}$ & $40 \%-50 \mathrm{mmHg}$ \\
L1 - left mainstem bronchus level 1 & $90 \%-20 \mathrm{mmHg}$ & $0 \%-50 \mathrm{mmHg}$ \\
R2 - right mainstem bronchus level 2 & $90 \%-10 \mathrm{mmHg}$ & \\
R1 - right mainstem bronchus level 1 & & \\
Carina & & \\
T3 - trachea level 3 & \\
T2 - trachea level 2 & $100 \%-10 \mathrm{mmHg}$ & \\
T1 - trachea level 1 & & \\
\hline
\end{tabular}

Epidemiology and classification 1 out of 2000 children can have a variable degree of tracheobronchomalacia, clinical diagnosis may be difficult in isolated cases, however severe cases are usually associated with syndromes such as tracheoesophageal, aortopulmonary malformations and bronchopulmonary dysplasia (1). Congenital tracheomalacia is produced by an alteration during the anterior intestine separation in the embryonic development of the trachea and esophagus. If there is also a tracheoesophageal fistula, a collapse zone will be located at this point. About $50 \%$ of the cases have cardiopulmonary defects associated (2). Acquired tracheomalacia occurs due to a compression that weakens the wall. Complex cardiopathies, mediastinal masses, abscesses and cystic lesions may also cause compression and ischemia on the wall. Iatrogenic lesions secondary to prolonged orotracheal intubation, tracheostomies, correction of esophagus and tracheal congenital defects may occur $(1,3)$.

\section{Clinical Presentation and diagnosis}

Most children present with symptoms such as stridor, cough and wheezing that can be misdiagnosed as bronchial hyperreactivity (1). Severe tracheomalacia shows signs of airway collapse as costal retractions, cyanosis and neck hyperextension (3). Young infants can have feeding difficulties due to compression from the alimentary bolus and brief resolved unexplained events (1). Airway evaluation through the 3- phase dynamic bronchoscopy is the gold standard. The first phase is done in a sedated patient maintaining spontaneous ventilation, which allows identification of the compressed areas, cartilaginous malformations, and excessive mucus accumulation. In the second phase cough is stimulated to reveal the maximal dynamic collapse. In the third phase, airway is filled with water to detect previous unidentified defects, such as tracheoesophageal fistula, tracheal diverticulum, and fixed compression areas (4). A collapse above $50 \%$ during cough or exhalation makes the tracheobronchomalacia diagnosis.

\section{Treatment}

The treatment for mild disease consists of symptom control, for moderate to severe disease the continuous positive airway pressure might be effective but uncomfortable for the patient and family (3). Surgical treatment is indicated in patients who do not respond to conservative treatment, patients with BRUE, recurrent pneumonia and repetitive orotracheal intubation (5). Aortopexy reduces anterior vascular compression of the trachea by elevating the aorta and fixating it to the posterior side of the sternum, tracheopexy pins up 
the posterior wall of the trachea to the anterior longitudinal ligament of the spine guided by bronchoscopy preventing airway collapse. (5).

\section{Anesthetic management}

As bronchoscopy is needed during the tracheopexy, airway management, ventilation mode and anesthetic management becomes a challenge for the anesthesiologist. Double lumen endotracheal tubes are not designed for neonates. The bronchial blockers are a great alternative for OLV, but they occupy a great space. An alternative is using the blocker from the outside and guide it through the main bronchi with the help of the fibre optic bronchoscope (3). Adjustments in ventilation mode will be made to oxygenate and ventilate adequately while allowing a good view of the surgical field. The wider lumen tube that fits the glottis should be selected to allow ventilation and bronchoscope introduction without causing an ischemic lesion of the tracheal mucosa. The ventilatory strategy must be able to maintain an adequate oxygenation, blood saturation above 88- $90 \%$ and an expected $\mathrm{CO} 2$ value, tolerating increases of $30 \%$ with respect to the basal during OLV or surgical manipulation of lung parenchyma and trachea. A CVC is recommended for hemodynamic support. An AL is useful due to the length of these procedures and fluid shift throughout the surgery. For a brachiocephalic trunk pexy, an AL in the right arm must be considered to evaluate the artery permeability and arm perfusion after fixation. Depth anesthesia monitoring helps with propofol titration doses. The anesthetic technique of choice is TIVA, as the open airway during the surgery will make inhaled anesthetics concentrations difficult to assess and achieve (3), on the other side, infusions allow adequate unconsciousness and analgesia. Neuromuscular relaxation allows lower doses of opioids and propofol improving ventilatory control, its use will be conditioned by neurofunctional monitoring of the Recurrent Laryngeal nerve. A multimodal analgesic strategy will allow early extubation.

\section{Learning points}

1. ventilation modes are modifiable throughout procedures

2. learning skills in flexible bronchoscope is key and useful in airway procedures

3. assertive and continuous communication amongst the surgical team determines patient outcomes

\section{ACKNOWLEDGEMENTS}

No source of funding to declare.

\section{CONFLICT OF INTEREST}

No conflict of interest to declare.

\section{References}

1. Boogaard R, Huijsmans SH, Pijnenburg MW, Tiddens HA, de Jongste JC, Merkus PJ. Tracheomalacia and bronchomalacia in children: incidence and patient characteristics. Chest. 2005;128:3391- 3397.

2. Fraga JC, Jennings RW, Kim PC. Pediatric tracheomalacia. Semin Pediatr Surg. 2016;25:156-164.

3. Masaracchia MM, Polaner DM, Prager JD, DeBoer EM, Dewberry LC, Somme S, Wine T, Janosy NR. Pediatric tracheomalacia and the perioperative anesthetic management of thoracoscopic posterior tracheopexy. Paediatr Anaesth. 2018;28:768-773.

4. Kamran A and Jennings RW (2019) Tracheomalacia and Tracheobronchomalacia in Pediatrics: An Overview of Evaluation, Medical Management, and Surgical Treatment. Front. Pediatr. 7:512.

5. Carden KA, Boiselle PM, Waltz DA, Ernst A. Tracheomalacia and tracheobronchomalacia in children and adults: an in-depth review. Chest. 2005;127:984-1005 

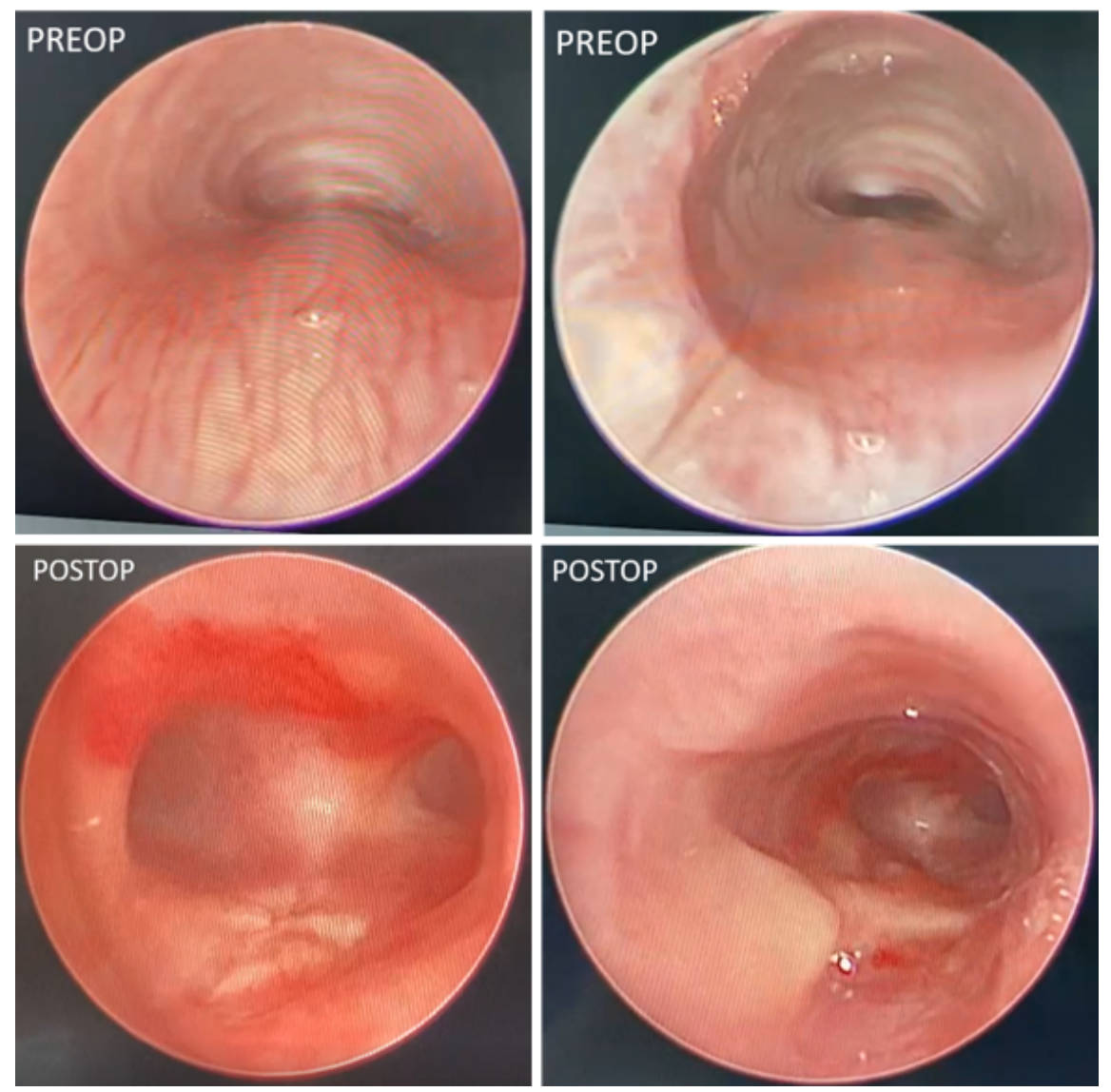

\section{Hosted file}

SHALLOW BREATHING.docx available at https://authorea.com/users/460666/articles/556569anesthetic-management-of-aortopexy-posterior-tracheopexy-and-esophageal-atresia-repairin-a-preterm-newborn 